\title{
LIQUID IONIZATION CALORIMETRY: REVIEW AND PREVIEW
}

\author{
C.W. Fabjan \\ CERN, Geneva, Switzerland . \\ CERN LIBRARIES, GENEVA

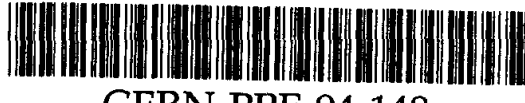 \\ CERN-PPE-94-148
}

\begin{abstract}
The experimental requirements at existing and planned accelerators, and new facilities for underground or space-borne experimentation have stimulated a wide-ranging $R \& D$ programme in liquid ionization calorimetry. Precision sampling calorimetry is approaching 'crystal' energy resolution whilst their rate capabilities will be able to cope with the highest LHC luminosities. (Quasi)-homogeneous noble-liquid calorimeters are under construction or in the planning stage to address some of the most fundamental physics questions.
\end{abstract}

Presented at 'Frontier Detectors for Frontier Physics, 6th Pisa Meeting on Advanced Detectors, La Biodola, May 1994. 
During the past five years, two advances have contributed in significant ways to the developments in Liquid Ionization Calorimetry. One advance is of rather fundamental character, applicable to all of calorimetry. It concerns the insight, verified experimentally in various calorimeter systems, that the performance of sampling calorimeters depends only weakly on the orientation of the absorber plates and active sensors (licuid, scintillator, etc.). While this perception is relatively old, imaginative applications of this principle have made a considerable impact on practical instruments. The second achievement is the 'mastery' of the cryogenic liquids for-sometimes very large--homogeneous calorimeters which achieve 'crystal-calorimetry' energy resolution and 'bubble-chamber' quality of event detail and reconstruction.

Applications exemplifying these two principles will be discussed in the following sections.

\section{PRECISION ELECTROMAGNETIC SAMPLING CALORIMETRY}

The 'accordion'-concept of absorber plate orientation (Fig. 1) is probably the most prominent illustration of the principle of 'performance-independence of absorber orientation'. It was applied previously to the orientation of pressurized gas ionization chambers to instrument magnet yokes of large spectromer magnets [1]. The same idea was also extensively used in scintillating fibre calorimetry $[2,3]$. In an imaginative variation on this theme scintillating tiles (and absorber plates) are oriented along the shower direction, a concept under construction for the large ATLAS hadronic barrel calorimeter [4].

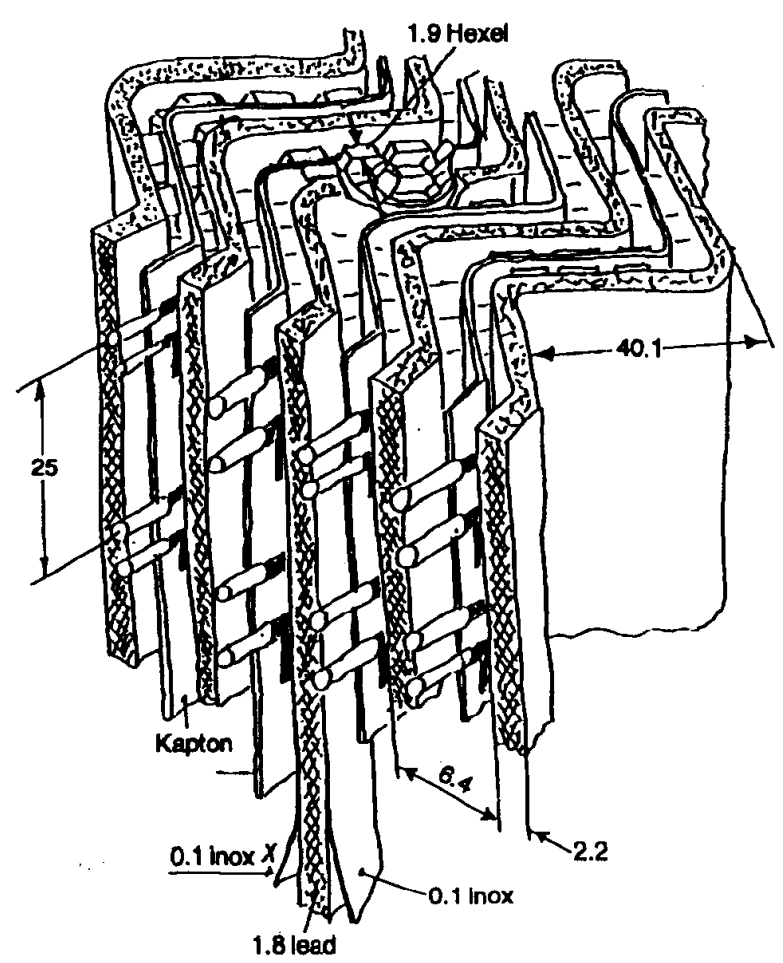

Figure 1: An 'artist's' rendering of the accordion absorber and readout structure. Metal electrodes (e.g. stainless-steel clad lead plates) are interleaved with thin copper electrodes on a Kapton support. The construction permits the connection of readout electronics at the entrance and exit face of the calorimeter. 
The accordion concept, was invented in response to the unusually demanding performance requirements of electromagnetic calorimetry at the LHC (and SSC) [5]:

- excellent energy resolution $(<0.1 / \sqrt{E})$ combined with a very small constant term $(\lesssim 0.5 \%)$

- very high segmentation in 'towers' combined with excellent hermeticity;

- fast charge transfer (i.e. short signal cables) to achieve the needed fast time response in the high-rate LHC environment.

The construction adopted (Fig. 1) satisfies all these requirements. While the absorber plates can be arranged (at a certain price of complexity) to hermetically fill the volume of a cylindrical calorimeter, the printed-circuit type kapton-copper electrodes can easily be segmented in rather complex ways to achieve the physics-imposed segmentation.

Rapid progress in this technology has culminated in the construction and test of a large prototype, a fair replica of a sector of the future ATLAS e.m. calorimeter (Fig. 2). The following construction features have been implemented [6]:

- the thickness of the Pb-absorbers was decreased from $\mathrm{d}=1.8 \mathrm{~mm}$ to $\mathrm{d}=1.2 \mathrm{~mm}$ for $\eta>0.7$. This change maintains the sampling term at a level adequate for the energy resolution target;

- the towers project towards the collision point and have a three-fold longitudinal segmentation. It permits good spatial and angular resolution;

- for best energy resolution the liquid-argon gap is maintained constant in depth: the accordion bend angle has to be varied from $87^{\circ}$ at the innes radius to $111^{\circ}$ at the outer.

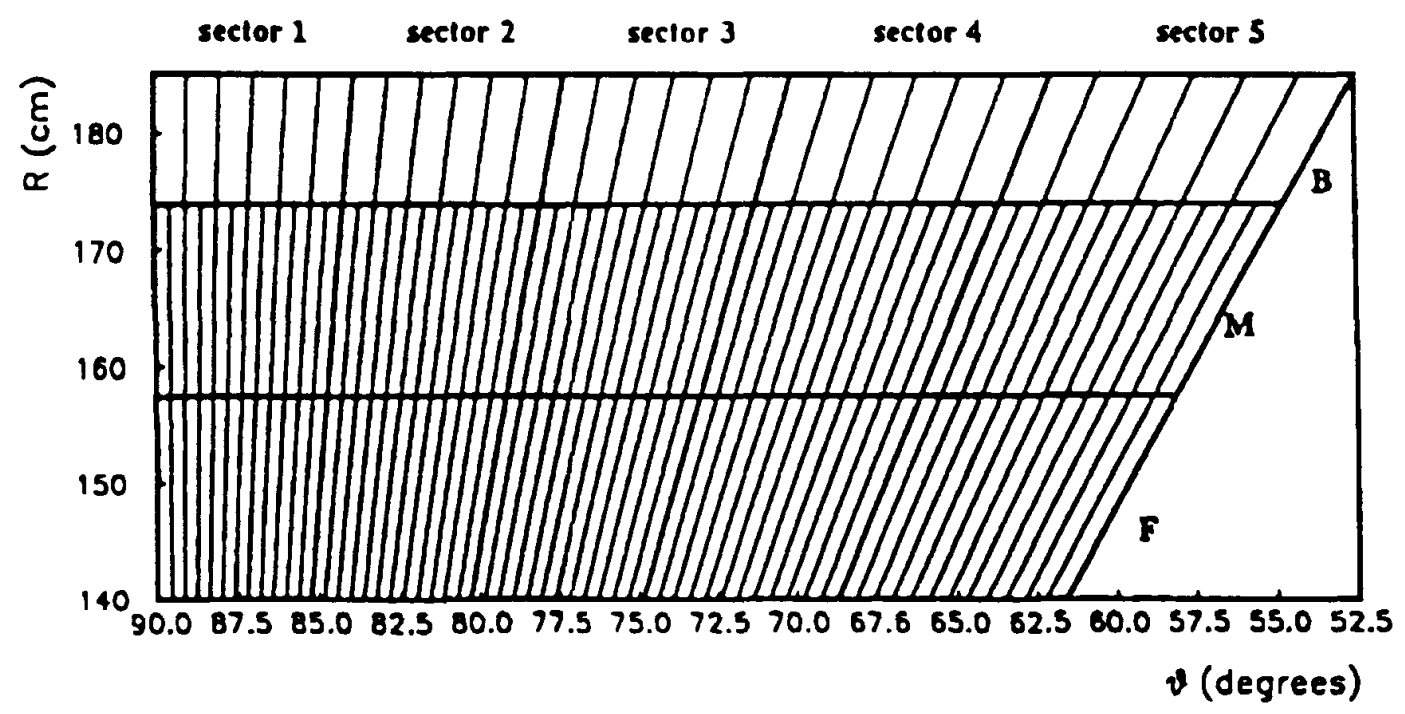

Figure 2: Schematic segmentation of the ' 2 metre' accordion prototype. The device extends over $27.5^{\circ}$ in polar angle and $30^{\circ}$ in azimuth.

In the quest, for very high energy resolution, an even more complex electrode arrangement was tested by the GEM Collaboration [7], see Fig. 3. It results in a denser construction or-at same average density -- in a reduced sampling term and hence better resolution. 


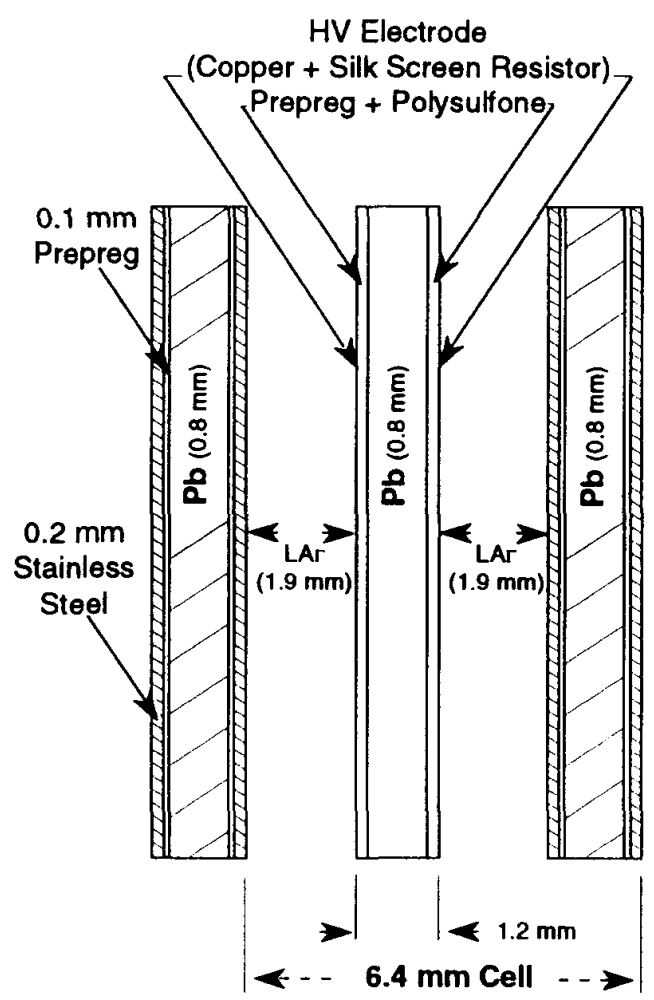

Figure 3: Electrode construction with all-absorber electrodes. The scheme achieves denser construction and/or reduced sampling fluctuations.

Test results of the $2 \mathrm{~m}$ prototype are summarized in Figs. 4 and 5 . The local uniformity (Fig. 4) shows an r.m.s. variation of $\sim 0.5 \%$. This regular $\phi$-modulation is a reflection of the accordion geometry and can be reduced by a global correction. The global uniformity over a large part of the prototype (Fig. 5) is measured to be r.m.s. $=0.58 \%$.

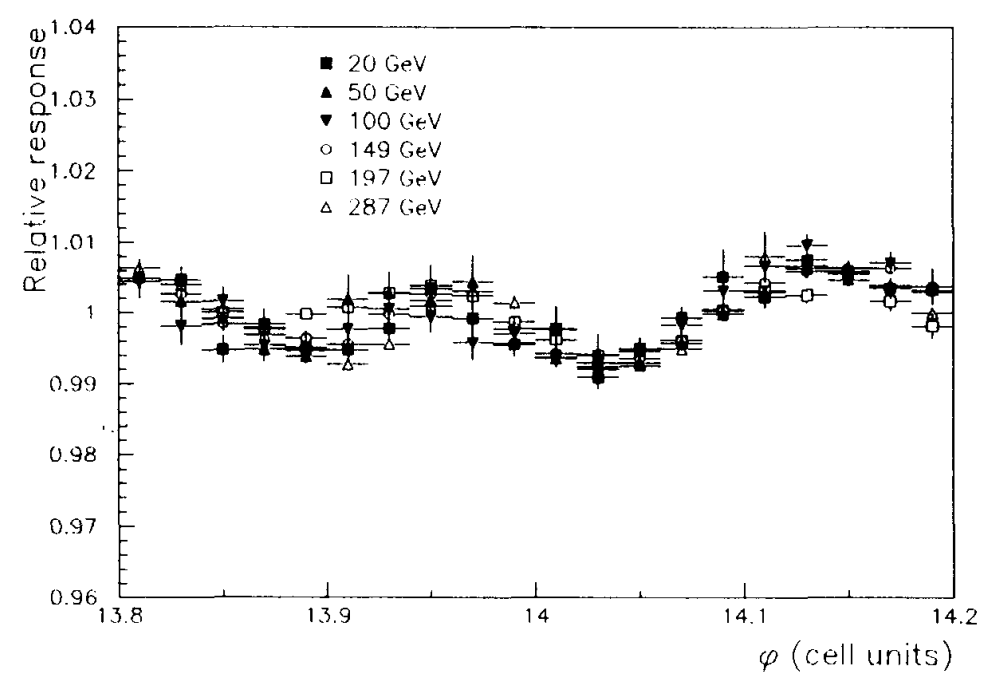

Figure 4: The local uniformity of the cell response measured in the $2 \mathrm{~m}$ prototype. 


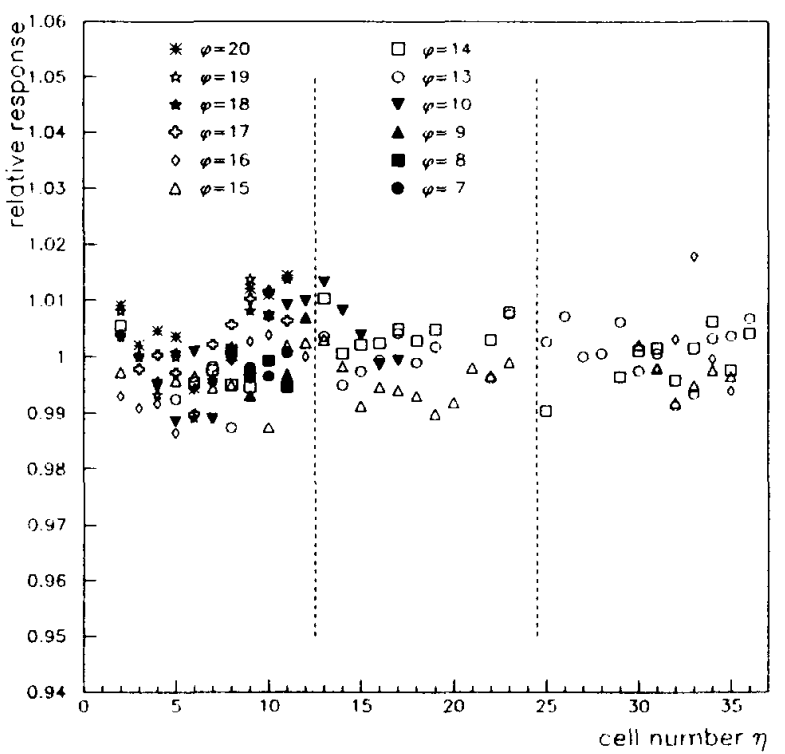

Figure 5: The uniformity measured with $287 \mathrm{GeV}$ electrons scanning the active surface of the $2 \mathrm{~m}$ prototype.

The energy resolution for this device, parametrized as $\sigma(E) / E=a / \sqrt{E} \oplus b / E \oplus c$ is given in Table 1.

Table 1: Energy resolution of the 2-m prototype

\begin{tabular}{|l|c|c|}
\hline & $\eta=0.28$ & $\eta=0.90$ \\
\hline $\mathrm{a}(\% \mathrm{GeV}(1 / 2)$ & $9.99 \pm 0.29$ & $10.24 \pm 0.33$ \\
$\mathrm{~b}(\mathrm{MeV})$ & $282 \pm 17$ & $386 \pm 16$ \\
\hline
\end{tabular}

The constant term $\mathrm{c}$ was measured to be $\mathrm{c} \approx 0.7 \%$. Perhaps more significantly is the level of understanding achieved for the constant term c. The contributions, due to electronics and mechanics imperfections, are tabulated in Table 2.

Table 2: Contributions to the constant term of the 2 in prototype

\begin{tabular}{|l|c|}
\hline \multicolumn{1}{|c|}{ Effect } & Contribution (\%) \\
\hline Calibration & 0.2 \\
uniformity of calibration pulse & 0.15 \\
ADC channel $\rightarrow \mathrm{GeV}$ conversion & 0.2 \\
timing precision & 0.1 \\
residual cross-talk effect & 0.1 \\
cross-talk in the injection networks & \\
\hline Mechanics & 0.3 \\
residual modulation $(\phi)$ & 0.15 \\
gap uniformity & 0.3 \\
absorber thickness & 0.57 \\
\hline total $(\oplus)$ & \\
\hline
\end{tabular}

The results of the GEM tests [8] are quoted in Table 3. This group has measured calorimeters operated both with LAr and LKr, for which a significant improvement in energy resolution was observed. 
Table 3: Measured and Monte Carlo estimates [8] for several calorimeter sampling geometrics $(\eta=0)$

\begin{tabular}{|l|l|l|l|}
\hline GEM tests & Pb thickness & $\begin{array}{l}\text { LAr (Geant) } \\
\mathrm{a}\left(\% \mathrm{GeV}^{1 / 2}\right)\end{array}$ & $\begin{array}{l}\text { LKr (Geant) } \\
\mathrm{a}\left(\% \mathrm{GeV}^{1 / 2}\right)\end{array}$ \\
\hline BNL A & $1.3 \mathrm{~mm}$ & $7.7(7.7)$ & $6.7(6.7)$ \\
BNL B & $2 \times 0.8 \mathrm{~mm}$ & $6.3(6.2)$ & $(5.7)$ \\
GEM & $1 \mathrm{~mm}$ & $(6.3)$ & $(5.2)$ \\
\hline
\end{tabular}

Besides excellent energy and space resolution a stringent performance requirement on angular resolution (e.g. for the study Higgs $\rightarrow \gamma \gamma$ ) must be met. This is achieved using the longitudinal subdivision, sometimes in combination with a 'preshower' device. Angular resolutions in the range $\sigma_{\theta}=(30$ to 50$) \mathrm{mrad} / \sqrt{E}$ are typically obtained [9].

Electromagnetic and hadronic calorimeters are known to have rather good time resolution. This feature is of critical importance for LHC applications as the correct bunch crossing association of the various energy deposits is of course a necessity.

The 'world record' in timing resolution belongs probably to the KLOE Collaboration [10]. Measurements in the scintillating fibre/learl calorimeter indicated $\sigma_{t} \sim 40 \mathrm{ps}$ for 1 $\mathrm{GeV}$ energy deposits!

The GEM collaboration has carried out extensive timing studies based on 'time sampling' of the energy signals [11] (Fig. 6) as well as with constant fraction discrimination [12]. Results on timing resolutions are shown in Fig. 7. The measurements use the information from the energy deposit $\varepsilon_{i}$ in the front (f) and back (b) longitudinal sections. If weights $\omega_{i}$ are used

$$
\omega_{i}=1 /\left[\left(\frac{C i}{\varepsilon i}\right)^{2}+\sigma_{t, c u l}^{2}\right],
$$

the resolution is evaluated to be $\sigma_{T}^{2}=1 /\left[\omega_{f}+\omega_{b}\right]$, see Fig. 7. These studies indicated that the intrinsic shower fluctuations contribute $\sigma_{t} \sim 0.05 \mathrm{~ns}$; the dominant contribution was due to jitter in the sampling electronics. The studies demonstrated that for energy deposits as low as $\varepsilon \sim 100 \mathrm{MeV}$ timing with $\sigma \sim 4$ ns can be achieved, adequate for LHC operation.

The same studies also provided information on the energy resolution as a function of the amplitude sampling frequency. Results for various sampling patterns (111.. means sampling every $18 \mathrm{~ns}, 10101 \ldots$ every $36 \mathrm{~ns}$ ) are summarized in Table 4. Two conclusions are evident:

- calculated and measured values for the resolution agree very well;

- sampling at $54 \mathrm{~ns}$ intervals is almost as good as sampling every $18 \mathrm{~ns}$.

Table 4: Calculated and measured resolution for several different sampling patterns

\begin{tabular}{|c|c|c|c|c|c|}
\hline \multirow{2}{*}{ Label } & & \multicolumn{2}{|c|}{ Front section } & \multicolumn{2}{c|}{ Back section } \\
\cline { 2 - 6 } & Pattern & $\begin{array}{c}\sigma_{A}(\mathrm{calc}) \\
(\mathrm{MeV})\end{array}$ & $\begin{array}{c}\sigma_{A} \text { (meas) } \\
(\mathrm{MeV})\end{array}$ & $\begin{array}{c}\sigma_{A} \text { (calc) } \\
(\mathrm{MeV})\end{array}$ & $\begin{array}{c}\sigma_{A} \text { (meas) } \\
(\mathrm{MeV})\end{array}$ \\
\hline$A$ & 111111111 & 21.5 & $21.2 \pm 0.3$ & 30.0 & $29.5 \pm 0.4$ \\
$B$ & 101010101 & 22.0 & $21.6 \pm 0.3$ & 30.4 & $30.4 \pm 0.5$ \\
$C$ & 010101010 & 24.8 & $24.1 \pm 0.4$ & 34.7 & $36.2 \pm 0.6$ \\
$D$ & 101010100 & 26.1 & $24.7 \pm 0.4$ & 36.5 & $35.9 \pm 0.6$ \\
$E$ & 001010100 & 28.6 & $26.6 \pm 0.4$ & 40.5 & $40.3 \pm 0.6$ \\
$F$ & 010010010 & 24.4 & $24.2 \pm 0.4$ & 33.7 & $34.6 \pm 0.5$ \\
\hline
\end{tabular}




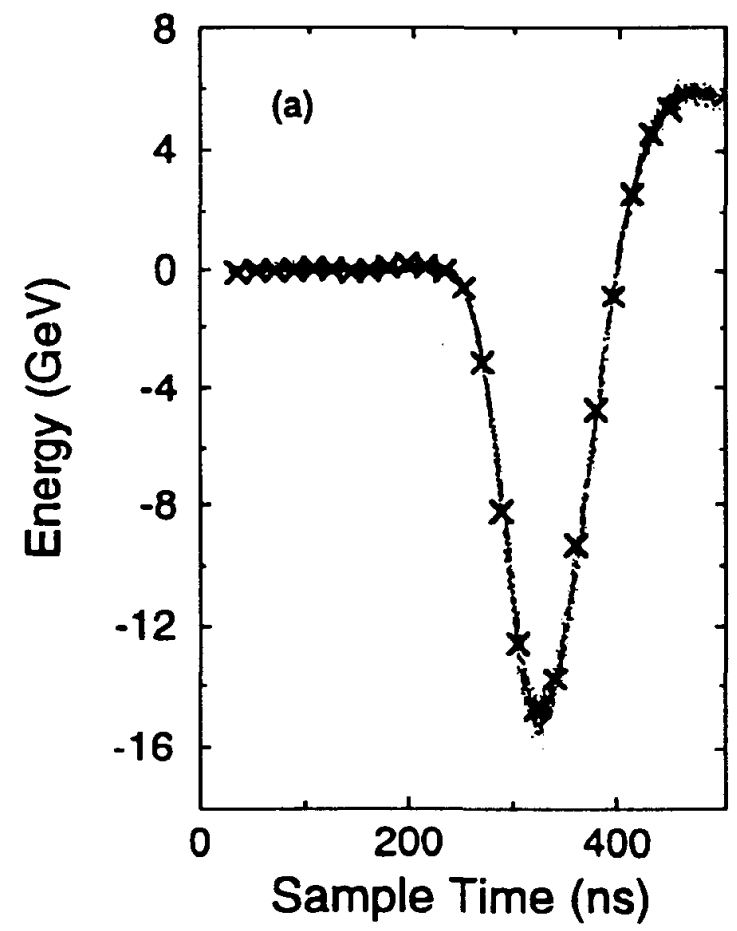

Figure 6: Time sampling of the energy signal obtained from $20 \mathrm{GeV} / \mathrm{c}$ electrons in a $5 \times 5$ tower matrix. The crosses on the wave form indicate the points of amplitude sampling.

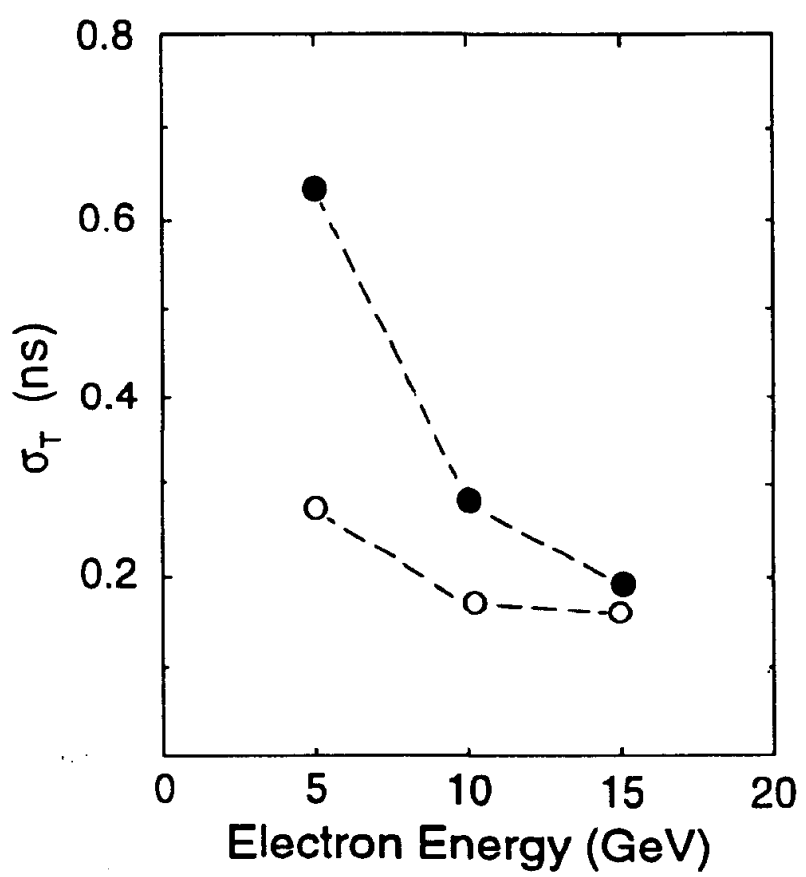

Figure 7: Results on timing resolution as a function of energy deposit. Considerable improvement is found for weighted results (open circles) as compared to unweighted values (solid circles). 
This progress on high-quality liquid calorimetry for LHC applications may be summarized in the following way:

- new absorber/electrode geometrics ('accordion') were developed to meet the LHC performance requirements;

- $\quad$ sampling calorimeters were developed with energy resolution in the $(0.06$ to 0.1$) / \sqrt{E}$ regime;

- large devices were constructed showing uniformity in response at the $0.5 \%$ level;

- the liquid ionization calorimeters show a good timing resolution of $\sigma \sim 4$ ns for $100 \mathrm{MeV}$ energy deposits;

- amplitude sampling of signals is understood and advantageous;

- fast, radiation hard, low power frontend electronics was developed, which can operate in the cryogenic liquids.

It is rewarding to conclude that the considerable work of many groups has produced all the required elements to build e.m. calorimeters which will meet the LHC performance requirements.

\section{3 (QUASI)-HOMOGENEOUS CALORIMETERS}

In this section we discuss a spectrum of calorimeter applications based on charge collection in a homogeneously sensitive cryogenic liquid (LAr, LKr, LXe). These applications aim at very high ('crystal-like') energy resolution, and also frequently complete event reconstruction, otherwise only achieved in bubble chambers or time projection chambers.

The NA48 Collaboration [13] is developing a very large, high-resolution homogeneous LKr-e.m. calorimeter. For the intended physics programme-CP-violation in the $K^{0}$-system-high resolution, high uniformity and a high rate capability are needed similar to the performance required ten years hence at the LHC. The parameters of this device and the energy resolution measured in a prototype are quoted in Table 5.

Table 5: Parameters and Performance of the NA48 LKr calorimeter

\begin{tabular}{|l|c|}
\hline Fiducial area & $4.5 \mathrm{~m}^{2}$ \\
Depth & $27 \mathrm{X}_{0}$ \\
Energy resolution measured & $\sigma / E=0.035 / \sqrt{E} \oplus \frac{40 \mathrm{MeV}}{E} \oplus 0.042 \%$ \\
Energy resolution expected & $\sigma / E \lesssim 0.035 / \sqrt{E}$ \\
Uniformity & $\lesssim 1 \%$ \\
Spatial resolution $[\mathrm{mm}]$ & $\sigma_{x} \approx 4.1 / \sqrt{E} \oplus 0.5$ \\
Tower size & $2 \times 2 \mathrm{~cm}^{2}$ \\
Number of channels & 13500 \\
\hline
\end{tabular}

The electrode structure is shown schematically in Fig. 8. The twin electrodes are stretched in the direction of the particle (shower) direction. The relatively large charge collection gap $(\sim 10 \mathrm{~mm})$ combined with the very fast pulse shaping ('initial current' measurement) makes this calorimeter structure very sensitive to small, geometrical imperfections in the charge-collecting structure: this was found to be a principal contribution to the resolution in early prototypes and has been reduced in the present design [13]. 


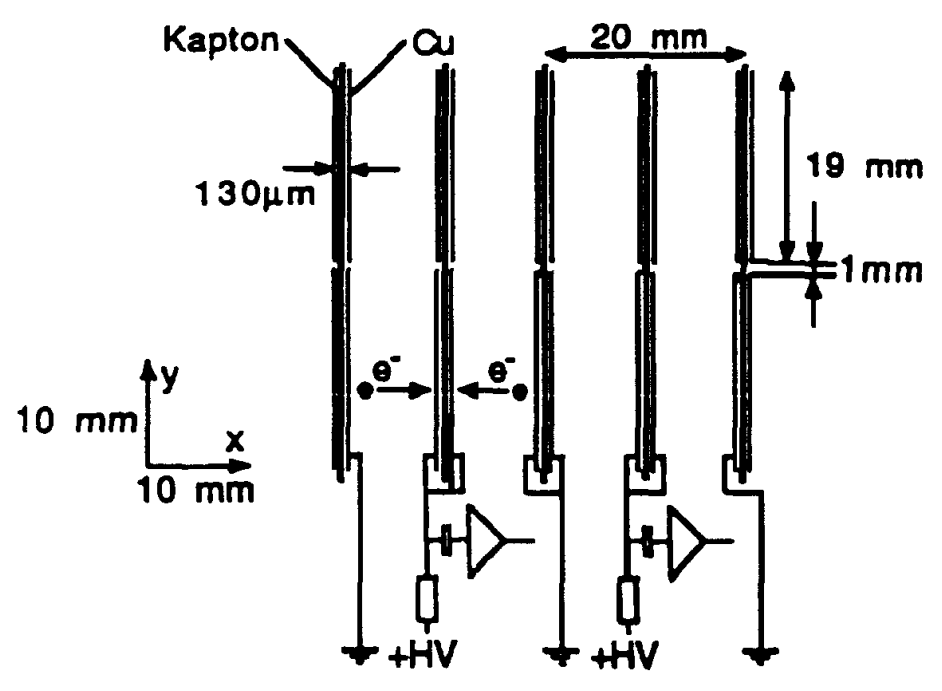

Figure 8: Electrode structure of the NA48 homogeneous LKr calorimeter. The electrodes are oriented in the direction of the incident particles.

The use of LKr was pioneered by the KEDR group working on VEPP-4M at Novosibirsk [14]. The principal motivations for the KEDR design were very good energy resolution, and good low energy $(E<500 \mathrm{MeV})$ performance combined with very good granularity. The electrode structure, built from Cu-clad fibre glass, is 'conventional' in orientation (fast response is not required): the electrodes are organized in $10 \times 10$ $\mathrm{cm}^{2}$ towers with threefold longitudinal subdivision; the first layer is divided into $5 \mathrm{~mm}$ wide strips to improve the spatial location of e.m. showers. Test-beam results confirm the expected, very good energy resolution:

$$
\begin{aligned}
\sigma_{E} & =6 \%(E=100 \mathrm{MeV}) \\
& =2 \%(E=1000 \mathrm{MeV})
\end{aligned}
$$

has been measured with a prototype [14]. The final calorimeter is under assembly.

The largest, homogeneous liquid ionization calorimeter is under development by the ICARUS Collaboration $[15,16]$. Its ultimate flight of fancy, using LAr, will take ICARUS below the Abruzzi mountains into the Gran Sasso Laboratory. Units of $\sim 4400 \mathrm{~m}^{3}$ each are planned to address the physics of proton decay, atmospheric and solar neutrino measurements, and possibly long baseline neutrino oscillation measurements. Critical for realizing the promise of such homogeneously sensitive detectors was the development of electronic readout schemes resulting in

- $\quad$ very good low-energy performance and good energy resolution $(\sigma / E \sim 3 \% / \sqrt{E(\mathrm{GeV})}$;

- high effective granularity $\left(\sim 2 \times 2 \times 2 \mathrm{~mm}^{3}\right)$, despite very long drift paths (up to $\sim 4 \mathrm{~m}$ ).

The rather complex electrode arrangements are sketched in Fig. 9. The superb pictorial reconstruction quality may be gleaned from the cosmic-ray shower, Fig. 10, reconstructed in a large prototype of 3 tonne fiducial mass. 


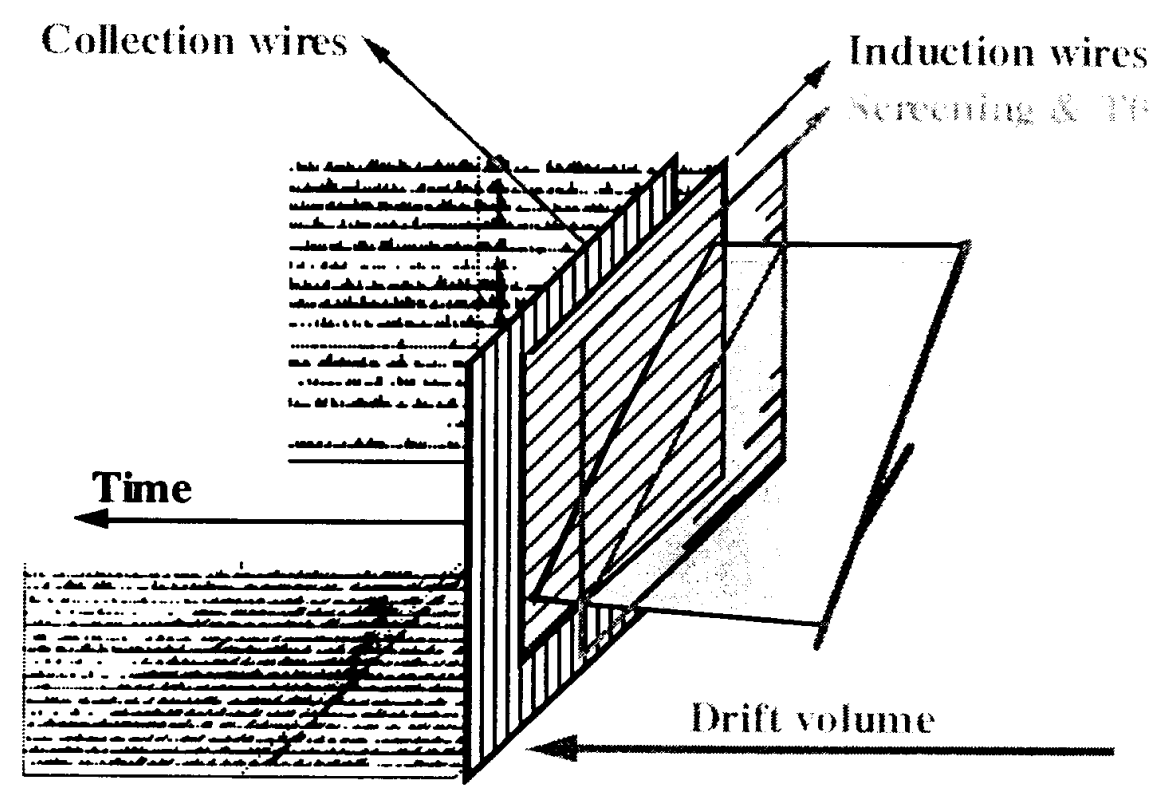

Figure 9: Conceptual arrangement of the collecting grids of ICARUS. Also shown is the collected charge on the time axis.

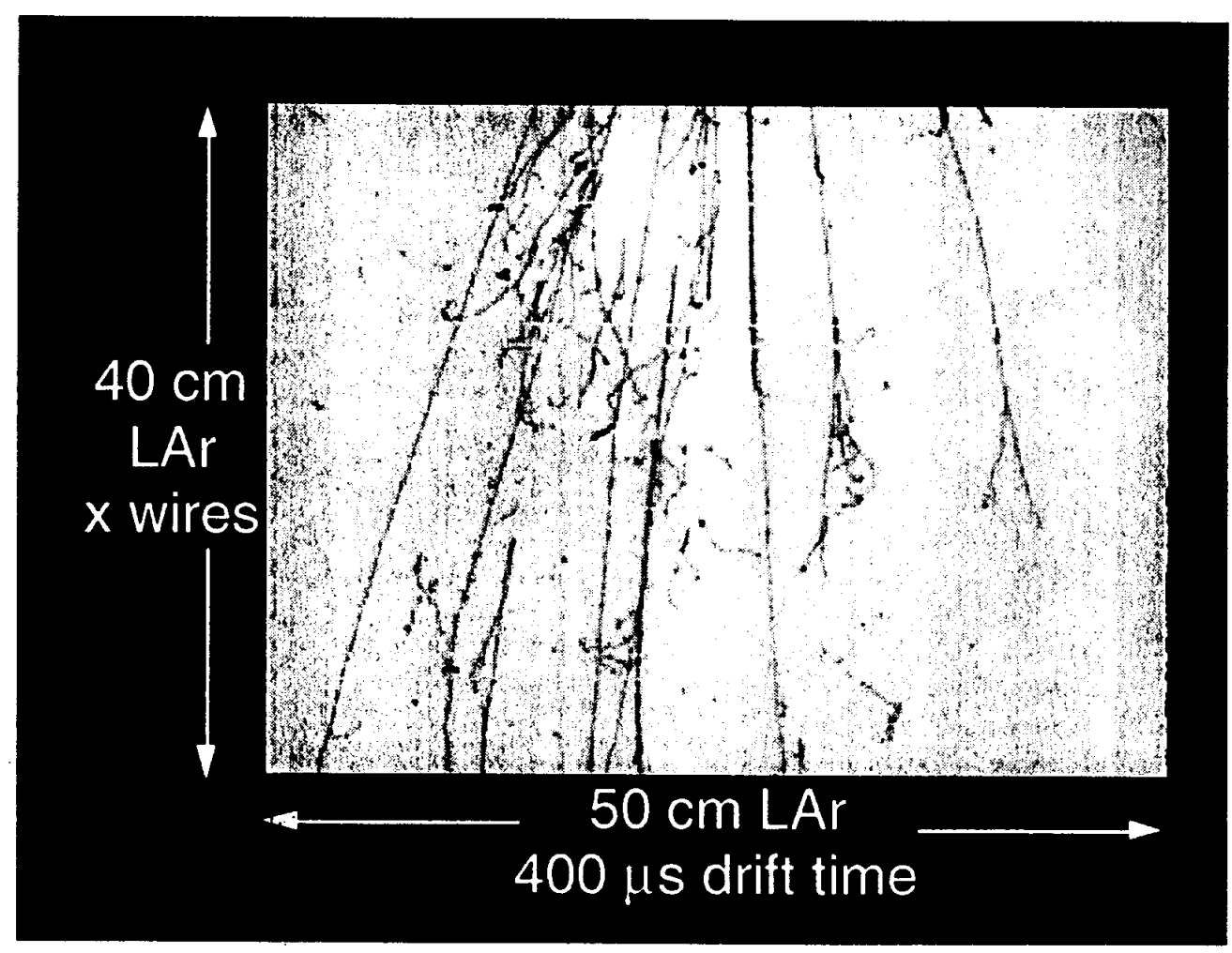

Figure 10: A cosmic-ray electromagnetic shower, reconstructed in the ICARUS prototype. 
Similar readout techniques, albeit for much smaller systems are being developed for LXe counters for astrophysics applications [17]. Such an instrument aims at imaging astrophysical $\gamma$-ray sources in the energy range of $\sim 100 \mathrm{keV}<E \lesssim 10 \mathrm{MeV}$. The key to a successful measurement is:

- very good energy resolution;

- very good directional and spatial resolution;

- background suppression with the aid of Compton kinematics.

The collection grids and electrode structure, rather similar to the ICARUS scheme, are shown in Fig. 11. The concept of using Compton kinematics for complete event reconstruction and the energy deposits of Compton and photoelectrons are shown in Fig. 12.

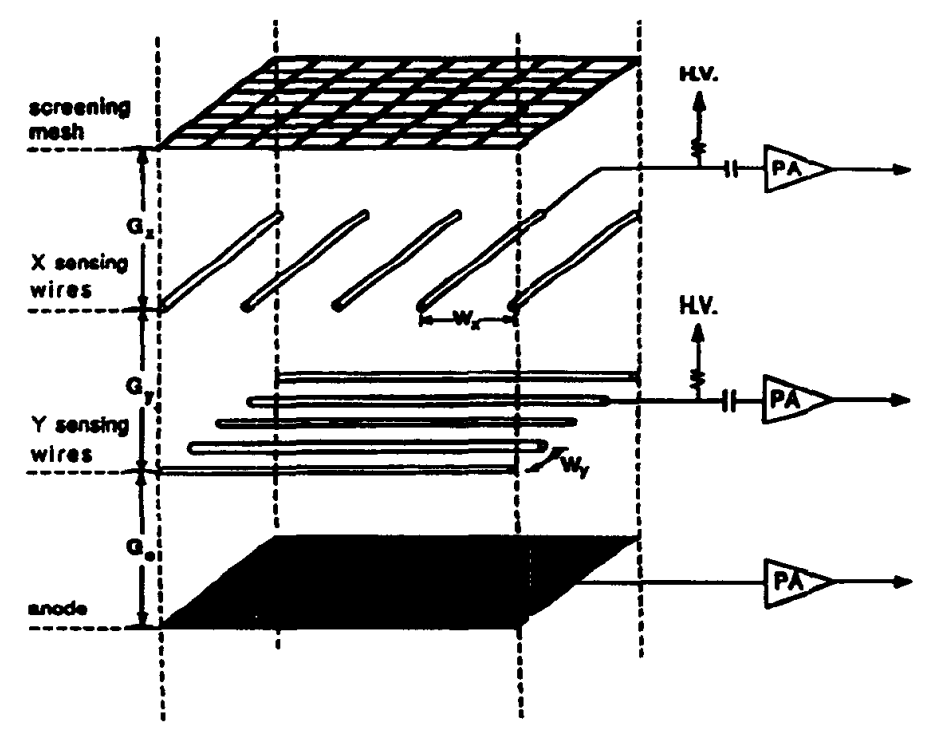

Figure 11: 3-dimensional readout developed for the LXe TPC. Wires, sensing the induced charge, provide two dimensions, whereas the drift-time provides information in the third direction.

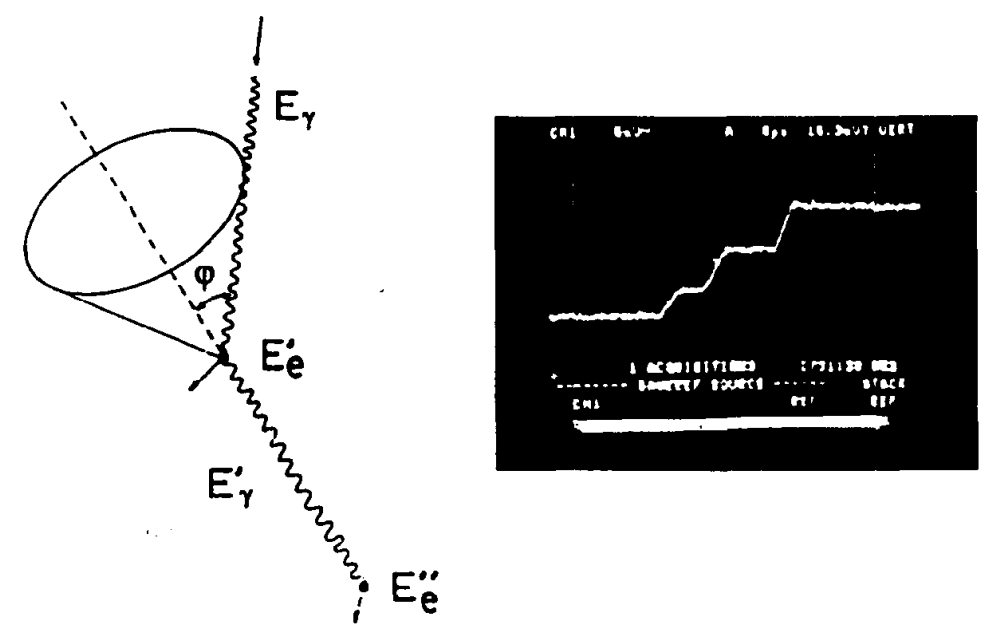

Figure 12: 'Compton kinematics' allows complete event reconstruction of the direction and energy of the incident $\gamma$-quantum, provided direction and energy of the Compton electron $\left(\mathrm{E}_{\mathrm{e}}^{\prime}\right)$ and the photoelectron $\left(\mathrm{E}_{\mathbf{e}}^{\prime \prime}\right)$ are measured. A scope picture shows the induced charge (= energy) of a multiple Compton-cum-photoelectron event. 
The scintillation light produced in the energy absorption process provides a 'natural' time marker for the drift measurement.

In laboratory tests a remarkable energy resolution of $\sigma / E \sim 2 \%$ at $1 \mathrm{MeV}$ was achieved, limited probably by recombination and hence not yet at the fundamental limit. In the same device a spatial resolution of $\sigma_{z} \sim 200 \mu \mathrm{m}$ and an angular resolution of $\sigma_{\theta} \sim 0.3^{\circ}$ (at $1 \mathrm{MeV}$ ) was observed.

As the next step, a LXe TPC is being readied for its maiden balloon flight. Will it open a new window in the electromagnetic spectrum of astrophysical observations?

A summary on homogeneous calorimetry would state:

- the purification of noble liquids has been mastered;

- the reward is 'crystal-like' energy resolution, demonstrated on rather large instruments;

- the development of ingenious readout methods provides, in addition, 3-dimensional event information;

- with these techniques assaults are under way on several frontiers of particle physics and particle astrophysics.

\section{LARGE LIQUID IONIZATION CALORIMER SYSTEMS}

Several experimental collaborations, working at the frontiers of high-energy physics, have adopted liquid ionization calorimetry. Some of the major systems are briefly described.

The D0 Collaboration, experimenting at the FNAL $1.8 \mathrm{TeV}$ p $\bar{p}$ collider, has constructed a high-quality combined electromagnetic and hadron calorimeter [18]. The group aimed for good hermeticity, good intrinsic compensation, and commensurate control of systematic effects.

These features are achieved with the parameters, as summarized in Table 6 .

Table 6: Major parameters of the D0 calorimeter

\begin{tabular}{|l|c|}
\hline Absorbes plates & $3 \mathrm{~mm}$ uranium \\
$\quad$ e.m. & $6 \mathrm{~mm}$ uranium \\
$\quad$ hadronic & $\Delta \phi \times \Delta \eta=0.1 \times 0.1$ \\
Granularity & $|\eta| \lesssim 4$ \\
Coverage & $\sigma / E \sim$ \\
Energy resolution & $\sigma / E=0.15 / \sqrt{E} \oplus 0.003$ \\
e.m. & $\sigma / E=0.5 / \sqrt{E} \oplus 0.004$ \\
hadronic (single particles) & $0.8 / \sqrt{E}$ \\
hadronic (jets) & $\sim 50000$ \\
hadronic (sampling) & $\sigma / E \sim 0.4 / \sqrt{E}$ (estimated) \\
Number of readout channels & $\sim 50$ \\
\hline
\end{tabular}

The calorimeter has operated reliably and stably. As an example the quality of LAr, monitored with $\alpha$-sources, is found to be stable to better than r.m.s. $<0.1 \%$ over the course of several years.

The most stringent demands on calorimeter performances are imposed by the HERA physics programme. The H1 Collaboration chose to develop an integrated e.m. and hadronic calorimeter, using lead and steel plates as absorber and LAr as the readout medium [19]. This calorimeter, intrinsically not compensated, can approach the performance of a compensated one using the method of 'off-line-compensation'. The longitudinal granularity 
(at least sevenfold segmentation) permits weighting as a function of the longitudinal energy deposit, as demonstrated in Fig. 13. The measured resolution parametrized in the form

$$
(\sigma / E)^{2}=a^{2} / E \oplus b^{2} / E^{2} \oplus c^{2}
$$

is characterized by [19]

$$
\begin{aligned}
& a=(50.7 \pm 0.7) \% \mathrm{GeV}^{1 / 2} \\
& b=945 \pm 32 \mathrm{MeV} \\
& c=1.6 \pm 0.1 \%
\end{aligned}
$$
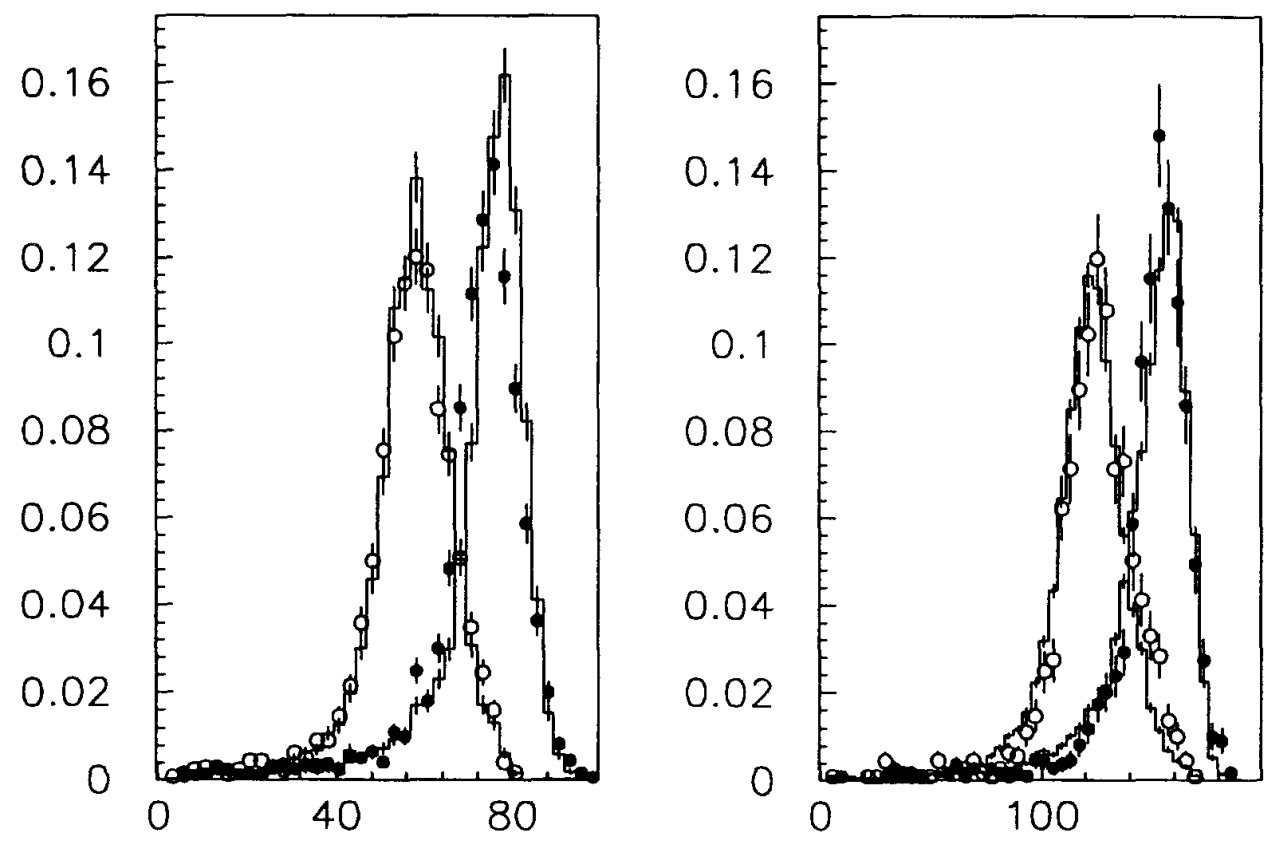

Figure 13: Total energy measured for pions at 80 and $170 \mathrm{GeV}$ in the $\mathrm{H} 1$ calorimeter. The open circles indicate the total signal and the full circles represent the signal after off-line longitudinal energy weighting.

The low constant term $c$ as well as the intrinsic resolution of $\sigma / E \sim 0.30 / \sqrt{E}$ (obtained from total energy resolution after deconvolution of the sampling fluctuations, estimated to contribute $\sigma / E \sim 0.4 / \sqrt{E}$ ) indicate that rather good off-line compensation has actually been achieved. The $\mathrm{H} 1$ collaboration could also demonstrate that this compensation scheme can also be applied to the measurement of jets [19].

The biggest LAr calorimeter in operation is BARS (Big Liquid Argon Spectrometer for Neutrino Physics), in the $\nu$-beam at Serpukhov [20]. The elementary readout cell is shown in Fig. 14, indicating that the 433 tonne LAr calorimeter is almost homogeneously sensitive. It has rather fine segmentation, using strips in the $x, u, v$ directions (total number $=27648)$. It is instructive to analyse the measured energy resolutions:

$$
\sigma / E(\text { e.m. })=0.04 / \sqrt{E} \oplus 0.08 / E \text {. }
$$

This 'crystal-like' resolution demonstrates very good control of systematic effects over this very large volume. 


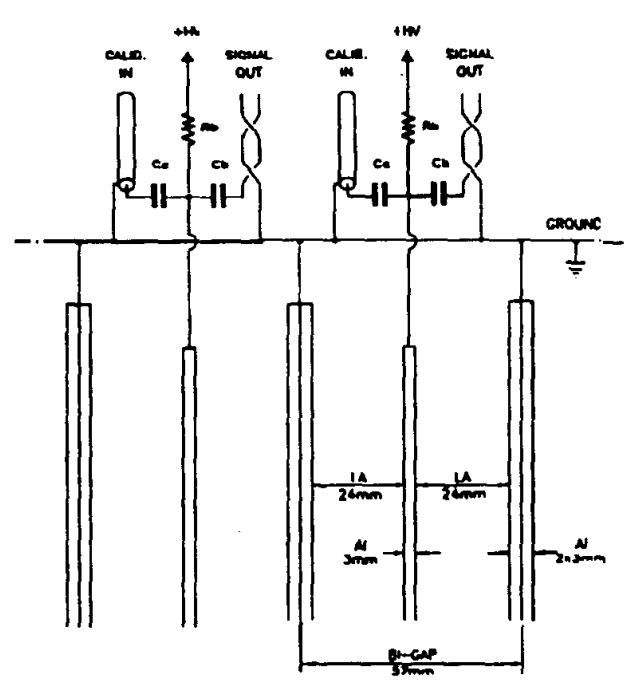

Figure 14: The readout all of BARS, indicating that this calorimeter is almost homogeneously sensitive.

The hadronic resolution is measured to be $\sigma / E$ (hadrons) $=0.55 / \sqrt{E} \oplus 0.02$. This resolution is typical for non-compensated calorimeters, even though this device is homogeneously active!

The total calorimeter, housed in two long cylindrical cryostats is an imposing sight: worth a trip!

This brief discussion of accelerator-based calorimeter systems would be incomplete without a mention of the preparations for the ATLAS calorimeter [21], schematically shown in Fig. 15. The e.m. calorimetry is based on 'accordion' geometries [6, 22]. The inner part of the end-cap hadron calorimeters will also use the LAr technology, whereas the extended outer cylinder will be built in the form of the novel scintillating tile calorimetry [4]. This hybrid solution was chosen to balance performance (e.m. resolution, radiation resistance) with cost.

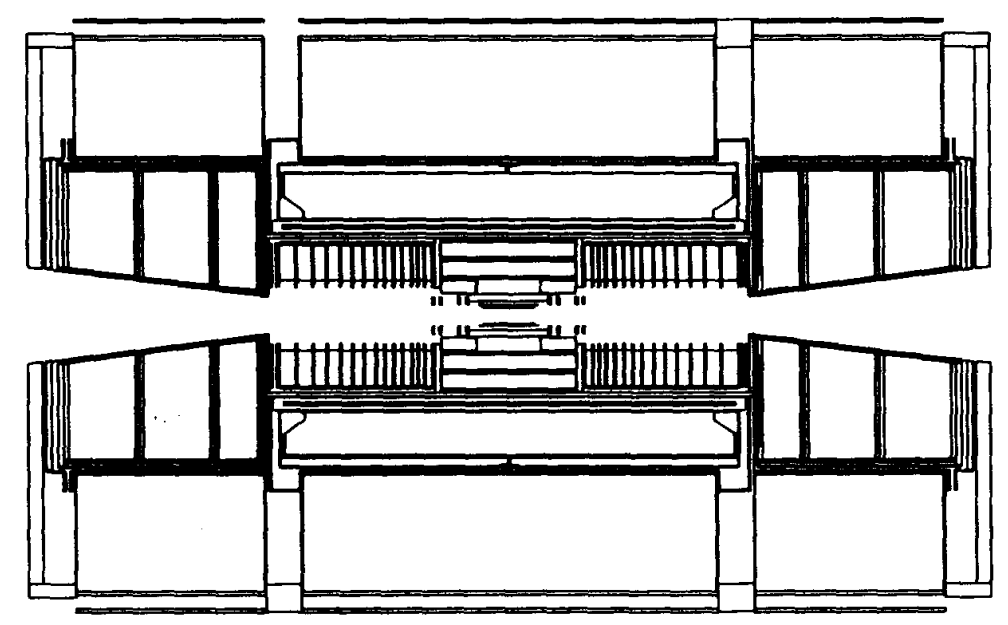

Figure 15: Cut through the planned ATLAS calorimeter and inner detector. The inner part of the calorimeter, extending to a radius of $r \sim 1.9 \mathrm{~m}$ uses LAr technologies. 


\section{Acknowledgements}

Many colleagues have provided timely information, which was much appreciated. The organizers graciously hosted a capacity crowd and arranged for a stimulating programme: La Biodela has become an Institution.

\section{References}

[1] V.I Baskakov et al. Nucl. Instrum. Methods 159 (1979) 83;

M. Barranco-Luque et al., BNL 31100 (1982)

[2] J. Badier et al., Nucl. Instr. Methods A337 (1994) 326.

[3] B. Bencheickh et al., Nucl. Instrum. Methods A315 (1992) 354.

[4] F. Arztizabal et al., CERN-PPE/94-66, to be published in Nucl. Instrum. Methods.

[5] B. Aubert et al., RD-3 Proposal, CERN/DRDC/90-32.

[6] D.M. Gingerich et al., Performance of a large scale prototype of the ATLAS accordion electromagnetic calorimeter, CERN-PPE/94, submitted to Nucl. Instrum. Methods.

[7] O. Benary et al., BNL 60272;

V. Radeka, Proc. IV International Conf. on Calorimetry in High Energy Physics, La Biodola, 1993.

[8] O. Benary et al., Nucl. Instrum. Methods A344 (1994) 363.

[9] B. Aubert et al., Nucl. Instrum. Methods A330 (1993) 405.

[10] S. Bertolucci et al., Proc. IV Int. Conf. on Calorimetry in High Energy Physics, La Biodola, 1993.

[11] O. Benary et al., BNL 60279, submitted to Nucl. Instrum. Methods.

[12] O. Benary et al., Nucl. Instrum. Methods A332 (1993) 78.

[13] V. Fanti et al., Nucl. Instrum. Methods (1994);

A. Ceccucci, contribution to his conference.

[14] V.M. Aülchenkov et al., Nucl. Instrum. Methods A316 (1992) 8;

Yu. Tikhomirov, private communication.

[15] P. Benetti et al., Nucl. Instrum. Methods A332 (1993) 395.

[16] P. Cennini et al., ICARUS proposal, Vol. II (1994).

[17] E. Aprile and M. Suzuki, IEEE Trans. NS36 (1989) 311;

E. Aprile et. al., Nucl. Instrum. Methods A333 (1993) 335;

E. Aprile et al., Proc. of the SPIE Conference (july 1994) and Columbia Univ., Astrophysics 549.

[18] S. Abachi et al., Nucl. Instrum. Methods A338 (1994) 185.

[19] B. Andrieu et al., Nucl. Instrum. Methods A336 (1993) 499.

[20] C. Cerri et al., Nucl. Instrum. Methods 227 (1989) 227;

F. Sergiampietri, Proc. IV Int. Conf. on Calorimetry, La Biodola, 1993.

[21] D. Gingrich et al., ATLAS Collaboration, Letter of Intent CERN/LHC/92-4 (1992).

[22] B. Aubert et al., RD-3 Status Report and further R\&D for calorimetry, CERN/DRDC/93-4 (1993). 